\title{
Serological measures to assess the efficacy of malaria control programme on Ambae Island, Vanuatu
}

Zulkarnain Md Idris ${ }^{1,2^{*}}$, Chim W. Chan ${ }^{1}$, Mubasher Mohammed', Morris Kalkoa ${ }^{3}$, George Taleo ${ }^{3}$, Klara Junker ${ }^{4,5}$, Bruno Arcà ${ }^{6}$, Chris Drakeley ${ }^{4}$ and Akira Kaneko ${ }^{1,7,8}$

\begin{abstract}
Background: Seroepidemiology can provide evidence for temporal changes in malaria transmission and is an important tool to evaluate the effectiveness of control interventions. During the early 2000s, Vanuatu experienced an acute increase in malaria incidence due to a lapse in funding for vector control. After the distribution of subsidised insecticide-treated nets (ITNs) resumed in 2003, malaria incidence decreased in the subsequent years. This study was conducted to find the serological evidence supporting the impact of ITN on exposure to Anopheles vector bites and parasite prevalence.

Methods: On Ambae Island, blood samples were collected from 231 and 282 individuals in 2003 and 2007, respectively. Parasite prevalence was determined by microscopy. Antibodies to three Plasmodium falciparum (PfSE, PfMSP-1 ${ }_{19}$, and PfAMA-1) and three Plasmodium vivax (PvSE, PvMSP-1 ${ }_{19}$, and PvAMA-1) antigens, as well as the Anopheles-specific salivary antigen gSG6, were detected by ELISA. Age-specific seroprevalence was analysed using a reverse catalytic modelling approach to estimate seroconversion rates (SCRs).

Results: Parasite rate decreased significantly $(P<0.001)$ from $19.0 \%$ in 2003 to $3.2 \%$ in 2007, with a shift from $P$. falciparum predominance to $P$. falciparum-P. vivax co-dominance. Significant $(P<0.001)$ decreases were observed in seroprevalence to all three $P$. falciparum antigens but only two of three $P$. vivax antigens (except PvAMA-1; $P=0$. 153), consistent with the more pronounced decrease in $P$. falciparum prevalence. Seroprevalence to gSG6 also decreased significantly $(P<0.001)$, suggesting that reduced exposure to vector bites was important to the decrease in parasite prevalence between 2003 and 2007. Analyses of age-specific seroprevalence showed a three-fold decrease in $P$. falciparum transmission, but the evidence for the decrease in $P$. vivax transmission was less clear.

Conclusions: Serological markers pointed to the effectiveness of ITNs in reducing malaria prevalence on Ambae Island between 2003 and 2007. The recombinant gSG6 antigen originally developed to indicate exposure to the Afrotropical vector An. gambiae may be used in the Pacific to complement the traditional measure of entomological inoculation rate (EIR).
\end{abstract}

Keywords: Malaria, Serology, Island, ITN, Plasmodium falciparum, Plasmodium vivax, Anopheles

\footnotetext{
*Correspondence: zulkarnain.mdidris@ki.se

${ }^{1}$ Island Malaria Group, Department of Microbiology, Tumor and Cell Biology,

Karolinska Institutet, Stockholm, Sweden

${ }^{2}$ Department of Parasitology and Medical Entomology, Faculty of Medicine,

Universiti Kebangsaan Malaysia Medical Centre, Kuala Lumpur, Malaysia

Full list of author information is available at the end of the article
} 


\section{Background}

In recent years, analyses of antibody responses to one or more malaria-specific antigens have been widely used to assess transmission intensity [1-3], temporal and spatial reductions in transmission [2, 4], as well as to confirm elimination $[5,6]$. In areas of low transmission, longlasting antibody responses may be easier to detect than parasite prevalence in human populations or infected mosquitoes [2], making serological measures useful adjunct metrics to measure malaria transmission. Recently, serological evidence of exposure to malaria has demonstrated successful interventions in some lowtransmission settings [7-9].

Located at the margin of malaria transmission, Vanuatu is an archipelago of more than 80 islands in the South Pacific. Malaria is endemic on most of the nation's 68 inhabited islands [10]. The majority of infections are due to Plasmodium falciparum and Plasmodium vivax, with some rare cases of Plasmodium malariae [10, 11]. There are two seasons in Vanuatu: the dry and cool season from May to October, and the wet and hot season from November to April. Hence malaria incidence in Vanuatu shows seasonal fluctuations, which are more pronounced for $P$. falciparum than $P$. vivax $[10,12]$. The only known malaria vector is Anopheles farauti (s.s.), a member of the Anopheles punctulatus complex [13].

Several large-scale control programmes have been implemented in Vanuatu over the past decades. In 1988, insecticide-treated nets (ITNs) were introduced; by 1992 ITN coverage had reached $27 \%$ of Vanuatu's population [10]. The sharp decline in malaria incidence during the 1990s was explained by the equitable ITN distribution that targeted the most vulnerable populations i.e. children under five years, their mothers, and pregnant women [12]. An integrated malaria elimination programme was initiated on the southern-most island of Aneityum in 1991 [14]. By the mid-1990s, with a high degree of commitment from the local communities, malaria had been eliminated from Aneityum [14, 15]. An acute increase in malaria incidence was observed after shortages in funding for malaria control in the early 2000s, followed by a decline in incidence after the resumption of distribution of subsidised ITNs. Since 2009, significant external support has enabled the expansion of intervention measures including the distribution of free long-lasting insecticide treated-bed nets (LLINs) to cover more than $80 \%$ of the population, focal indoor residual spraying (IRS), the introduction of free rapid diagnostic test (RDT) and free artemisinin-based combination therapy (ACT) to all health facilities, and enhanced surveillance and rapid response to identified cases. Further decrease in malaria incidence since 2009 has put Vanuatu on course to achieve malaria elimination in the near future [16].
This study reports on the parasitological and seroepidemiological results from samples collected on Ambae Island in 2003 and 2007. In addition to responses to antigens from $P$. falciparum and $P$. vivax, exposure to vector mosquito bites was also evaluated, with the aim to determine the effect of vector control on parasite prevalence. The three parasite antigens used in this study vary in immunogenicity. The Plasmodium crude schizont extract (SE) is multi-antigenic and has greater sensitivity to detect low residual transmission and changes in transmission intensity [7, 17]. The blood-stage antigen merozoite surface protein- $1_{19}$ (MSP$1_{19}$ ) exhibits moderate immunogenicity and has been useful for estimating malaria transmission in populations across a gradient of transmission intensity [1]. The blood-stage apical membrane antigen-1 (AMA-1) is highly immunogenic and induces long-lived immune responses. In moderate transmission settings, seroconversion occurs more rapidly for AMA-1 than MSP-1 19 [2, 7]. The An. gambiae salivary gland antigen (gSG6) showed strong immunogenicity among African populations in previous studies $[18,19]$. The short-lived antibodies against gSG6 appear to correlate with changes in Anopheles abundance [20], which is useful for evaluating vector control strategies [21].

\section{Methods}

\section{Study sites and sample collection}

Ambae Island $\left(398 \mathrm{~km}^{2}\right)$ is in Penama Province (Fig. 1) and has a population of 10,407 (Vanuatu National Census, 2009). Transmission of $P$. falciparum, $P$. vivax and $P$. malariae is ongoing, and the transmission intensity is characterised as meso-endemic [10, 22]. Samples were collected during cross-sectional malariometric surveys conducted in south Ambae in June $2003(n=231)$ and June $2007(n=282)$. Futuna Island $\left(11 \mathrm{~km}^{2}\right)$ is in Tafea Province (Fig. 1) and has a population of 535 (Vanuatu National Census, 2009). In contrast to Ambae, Futuna lies beyond the Buxton line, which defines the southeastern limit of anopheline mosquito breeding, thus has always been free of malaria [10, 14, 17, 23, 24]. Samples were collected in July $2011(n=392)$ and were included in the analyses of parasite prevalence and vector exposure only.

Informed consent was obtained from all participants. The consent procedure was witnessed by a third party (e.g. teacher, village chief), who also recorded the name of each participant as he/she enrolled in the survey. Gender and age were recorded for each participant. Axillary body temperature was determined using a digital thermometer (Terumo, New Jersey, US). Fever was defined as a temperature exceeding $37.5{ }^{\circ} \mathrm{C}$. Spleen size was assessed in children aged 12 years and younger according to Hackett's method by one investigator only 


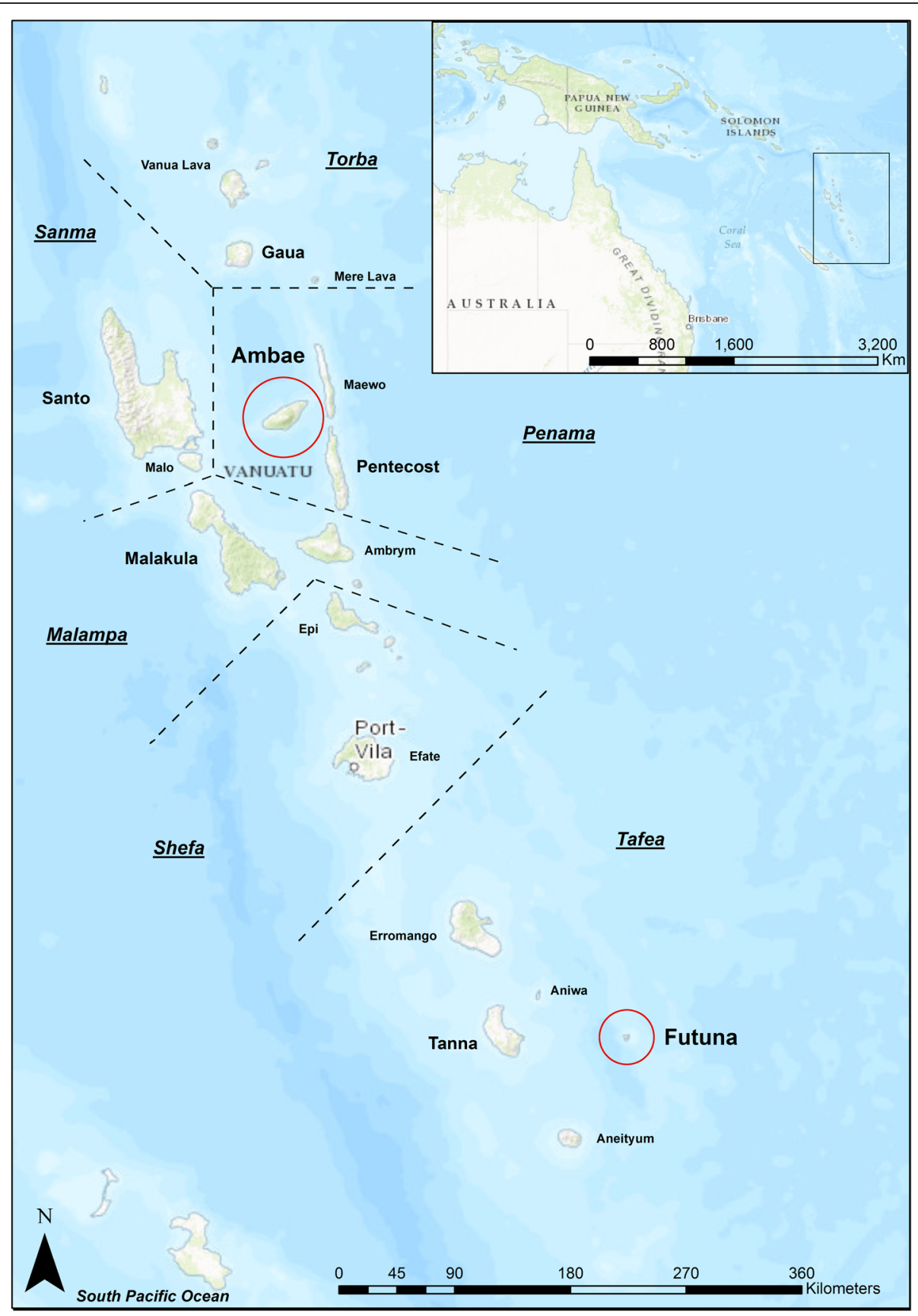

Fig. 1 Locations of Ambae Island and Futuna Island in Vanuatu. The names of the six provinces in Vanuatu are underlined, and approximate provincial boundaries are indicated by dashed lines. Inset shows the location of Vanuatu. The map was created with ArcGIS software, version 10.4, http://www.esri.com/

(AK). Blood samples were obtained for microscopic examination of malaria infections. Two spots of blood (70 $\mu$ l each) were collected on Whatman 31ET Chr filter paper (Whatman, Maidstone, UK). The blood spots were air-dried and stored in plastic bags at ambient temperature in the field and later at $-20{ }^{\circ} \mathrm{C}$ in our laboratories until processing. This study was approved by the Ministry of Health in Vanuatu and by the
Committee on the Ethics of Human Research of Karolinska Institutet in Sweden.

\section{Parasite prevalence by microscopy}

Thin and thick blood smears were stained with $3 \%$ Giemsa solution for $30 \mathrm{~min}$ and examined under oil immersion $(1,000 \times$ magnification) by experienced microscopists. Blood smears were defined as negative if no 
parasites were found after examining 100 high power microscopy fields. For all positive samples, malaria species were identified, and the presence of $P$. falciparum gametocytes was recorded.

\section{Assay of anti-malarial antibody responses}

A blood spot (3 $\mathrm{mm}$ in diameter) was punched from each sample and antibodies were eluted in reconstitution buffer in $0.5 \mathrm{ml}$ deep well plates (Corning Costar, PA, USA) as described previously [25]. The reconstituted blood spot solution, equivalent to a 1:200 dilution of serum, was stored at $4{ }^{\circ} \mathrm{C}$ until use.

All sera from Ambae were tested for IgG antibodies by indirect quantitative enzyme-linked immunosorbent assay (ELISA) to crude schizont extract (SE), the $19 \mathrm{kDa}$ fragment of recombinant merozoite surface antigen-1 (MSP-1 $\left.1_{19}\right)$, and recombinant apical membrane antigen-1 (AMA-1) for P. falciparum (PfSE, PfMSP-1 19 [Wellcome strain], and PfAMA-1 [3D7 strain]) and P. vivax (PvSE, PvMSP-1 19 [Belem strain], and PvAMA-1 [Sal-1 strain]) as described previously [25]. Crude SE of $P$. falciparum and $P$. vivax antigens were prepared as previously described [26, 27]. Briefly, NUNC-Immuno plates (Sigma-Aldrich, St. Louis, USA) were coated with $50 \mu$ l of coating buffer containing SE, AMA-1 and MSP- $1_{19}$ at $0.5 \mu \mathrm{g} / \mathrm{ml}$. The plates were washed in PBS with $0.05 \%$ Tween 20 (PBS/T) and blocked using 1\% (w/v) skimmed milk (Sigma-Aldrich) in PBS/T for three hours. After washing, $50 \mu \mathrm{l}$ of reconstituted serum were added in duplicate. The final antigen dilutions were 1:1000 for SE and MSP-1 19 , and 1:2000 for AMA-1. In addition, four blank wells and a five-fold dilution series of an African hyper-immune serum pool $(n=12)$ were added per plate. The plates were washed and $50 \mu \mathrm{l}$ of horseradish peroxidase (HRP)-conjugated rabbit antihuman IgG antibody (DAKO, Glostrup, Denmark) was added at a dilution of 1:15,000 in PBS/T and incubated for $3 \mathrm{~h}$. After further series of washes, antibody responses were detected after development with $100 \mu \mathrm{l}$ of the substrate solution 3, 3', 5, 5'-Tetramethylbenzidine (TMB) (tebu-bio laboratories, Le Perrey-en-Yvelines, France) for $15 \mathrm{~min}$. The reaction was stopped with $50 \mu \mathrm{l}$ of $2 \mathrm{M} \mathrm{H}_{2} \mathrm{SO}_{4}$. The optical density was read using Multiskan Go ELISA reader (Thermo Fisher Scientific, Waltham MA, USA) at $450 \mathrm{~nm}$.

All samples from Ambae and Futuna were tested for exposure to bites of An. farauti (s.s.) using the recombinant An. gambiae salivary gland gSG6 antigen [19]. ELISA was performed as described above for the parasite antigens, with the exception that NUNC-Immuno plates (Sigma-Aldrich) were coated with the recombinant antigen at $5 \mu \mathrm{g} / \mathrm{ml}$ in $50 \mu \mathrm{l}$ of coating buffer (final antigen dilution of 1:100).

\section{Statistical methods}

Data were double-entered and imported in STATA/SE version 13.1 (StataCorp, TX, USA). Optical density (OD) values were averaged and normalised against values from blank wells to adjust for background reactivity as previously described [25]. Seropositivity was determined by fitting a mixture model to normalised OD values assuming two Gaussian distributions, one for seronegative individuals and another for seropositive individuals [3]. The mean OD plus three standard deviations associated with the seronegative group was used as the cut-off value for seropositivity. A separate cut-off was generated for each antigen. Differences in proportions were tested using the Chi-squared test or the Fisher's exact test. Antibody levels among populations were compared using the Mann-Whitney U test or the Kruskal-Wallis test with Dunn's multiple comparison post-hoc tests. Pairwise correlations between ODs of different antibody responses were determined using the Spearman's rank correlation. Seroprevalence was stratified into yearly age groups and then analysed using a reverse catalytic modelling approach under a binomial sampling assumption, as described elsewhere $[1,9,28]$. This provides an estimate of the mean annual rates of conversion to seropositive (seroconversion rate, SCR $[\lambda]$ ) and reversion to seronegative (seroreversion rate, SRR $[\rho]$ ), averaged over the age of the population. Infants under 1 year of age were excluded to remove any influence of maternally derived antibodies [1]. Logistic regression was used to identify factors associated with seropositivity to any parasite-specific antigens and gSG6. In the regression analysis, seropositivity to Plasmodium was defined as being positive for either or both species-specific antigens (MSP-1 19 and/or AMA-1). Survey year, gender, age group, fever, and infection status were considered as explanatory variables in the univariate analyses. All variables with a $P$-value of $\leq 0.05$ from the likelihood ratio test in the univariate analyses were included in the multivariate logistic regression model. The variables included in the final (adjusted) model was survey year and age group.

\section{Results}

\section{Characteristics of the study population and parasite prevalence}

In total, 231 and 282 people were sampled from Ambae in 2003 and 2007, respectively; 392 people were recruited from Futuna in 2011. Most the sampled populations were 20 years old and younger. On Ambae, the gender ratio and age distribution of the samples did not differ between 2003 and 2007 (Table 1). The prevalence 
Table 1 Sample characteristics, clinical and parasitological results for all surveyed islands by year [\%, $(n)$ ]

\begin{tabular}{|c|c|c|c|c|c|c|}
\hline \multirow{3}{*}{ Characteristic } & \multirow{3}{*}{ Category } & \multicolumn{2}{|l|}{ Ambae } & \multirow{3}{*}{$P$-value ${ }^{a}$} & \multirow{3}{*}{$\begin{array}{l}\text { Futuna } \\
2011 \\
(n=392)\end{array}$} & \multirow{3}{*}{$P$-value } \\
\hline & & 2003 & 2007 & & & \\
\hline & & $(n=231)$ & $(n=282)$ & & & \\
\hline Gender & Female & $52.8(122)$ & $53.9(152)$ & 0.859 & $51.0(200)$ & 0.752 \\
\hline Median age $\left(\mathrm{IQR}^{\mathrm{C}}\right)$ & & $16(7-27)$ & $12(6-26)$ & 0.653 & $14(8-25)$ & 0.877 \\
\hline \multirow[t]{4}{*}{ Age group in years } & $0-5$ & $17.7(41)$ & $23.0(62)$ & 0.268 & $15.8(62)$ & 0.120 \\
\hline & $6-10$ & $22.5(52)$ & $20.2(57)$ & 0.588 & $18.9(74)$ & 0.552 \\
\hline & $11-20$ & $24.2(56)$ & $19.5(55)$ & 0.198 & $35.7(140)$ & $<0.001$ \\
\hline & $>20$ & $35.5(82)$ & $38.3(108)$ & 0.522 & $29.6(116)$ & 0.051 \\
\hline Fever $\left(>37.5^{\circ} \mathrm{C}\right)$ & & $8.2(19)$ & $0.0(0)$ & $<0.001$ & $8.2(32)$ & $<0.001$ \\
\hline Enlarged spleen ( $<12$ years old) & & $69.8(74 / 106)$ & $28.8(23 / 80)$ & $<0.001$ & $2.9(2 / 70)$ & $<0.001$ \\
\hline Parasite prevalence & & $19.0(44)$ & $3.2(9)$ & $<0.001$ & $0(0)$ & $<0.001$ \\
\hline \multirow[t]{3}{*}{ Species-specific prevalence } & P. falciparum & $11.7(27)$ & $1.8(5)$ & $<0.001$ & $0(0)$ & $<0.001$ \\
\hline & P. vivax & $6.5(15)$ & $1.4(4)$ & 0.004 & $0(0)$ & $<0.001$ \\
\hline & P. malariae & $0.9(2)$ & $0.0(0)$ & 0.204 & $0(0)$ & 0.054 \\
\hline Gametocyte prevalence $^{d}$ & P. falciparum & $7.8(18)$ & $0.7(2)$ & $<0.001$ & $0(0)$ & $<0.001$ \\
\hline
\end{tabular}

a Proportion or median level of each category between two surveys on Ambae Island (2003 and 2007)

b Proportion or median level of each category among all three surveys (Ambae Island in 2003 and 2007 and Futuna Island in 2011)

' IQR: interquartile range (25-75th percentile)

${ }^{\mathrm{d}}$ No $P$. vivax and $P$. malariae gametocytes were detected by microscopy

of fever, enlarged spleen in children, as well as Plasmodium infections were significantly lower in 2007 than in 2003 (Table 1; Chi-square test or Fisher's exact test: all $P<0.01)$. In both sampled years, most Plasmodium infections were found in children 10 years and younger (Fig. 3a). Plasmodium malariae was found in only two individuals in 2003, both of whom were co-infected with $P$. falciparum. The decrease in prevalence was more apparent for $P$. falciparum $(-9.9 \%)$ than $P$. vivax $(-5.1 \%)$, resulting in a shift in species composition from the predominance of $P$. falciparum in 2003 to the co-dominance of P. falciparum and $P$. vivax in 2007. The prevalence of $P$. falciparum gametocytes also decreased significantly
(Fisher's exact test: $P<0.001)$ between the sampled years (Table 1).

As expected, no malaria infection was detected on $\mathrm{Fu}$ tuna. The prevalence of enlarged spleen in children on Futuna was significantly lower (Fisher's exact test: both $P<0.001$ ) than that from Ambae in both 2003 and 2007. The prevalence of fever on Futuna was similar to that on Ambae in 2003 (Table 1). These fever cases were likely caused by other infections, but they were not explicitly investigated during our survey.

\section{Breadth and correlation of different antibody responses}

Antibody levels as measured in optical densities are shown in Fig. 2. The median anti-gSG6 antibody level
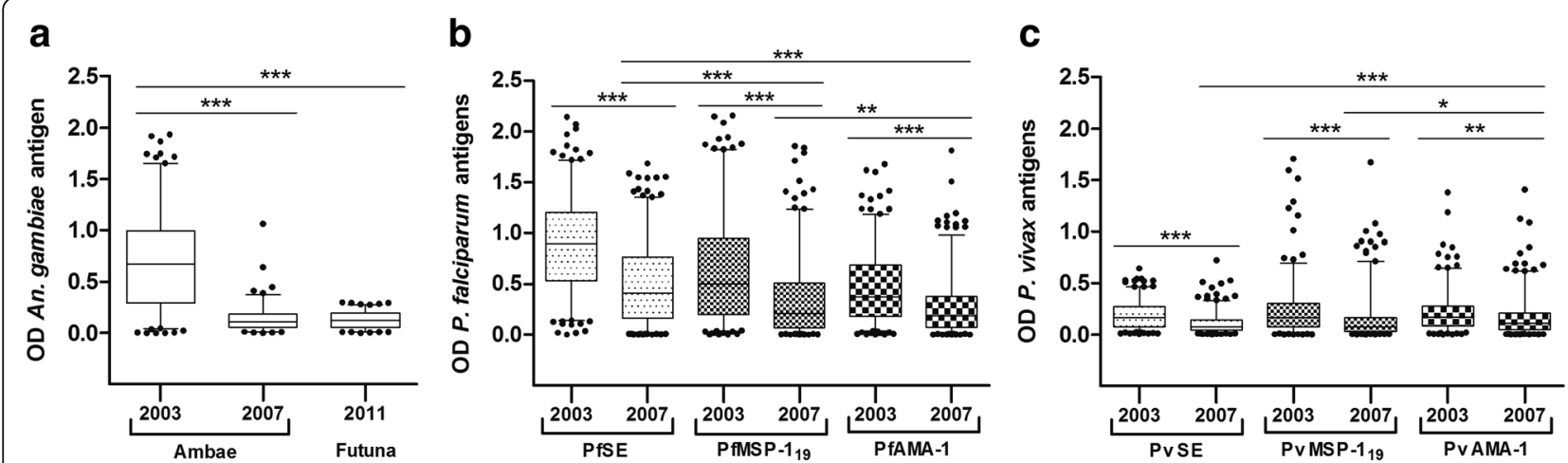

Fig. 2 Species-specific antibody responses to An. gambiae salivary gland gSG6 antigen (a), $P$. falciparum antigens (b), and $P$. vivax antigens (c). The interquartile range (IQR, 25-75th percentile) is represented in a box plot with the median OD value shown as a line within the box. ${ }^{*}<0.05$; ${ }^{* *} P<0.01 ;{ }^{* * *} P<0.001$ 
showed a significant (Mann-Whitney U-test: $Z=6.57, P$ $<0.001$ ) decrease on Ambae between 2003 and 2007. No significant difference in gSG6 antibody levels was observed between Ambae in 2007 and Futuna, where Anopheles vectors are absent. Similar to the decrease in anti-gSG6 antibody levels, median antibody levels to all $P$. falciparum and $P$. vivax antigens also decreased significantly (Kruskal-Wallis H-test: all $P<0.01$ ) on Ambae between the survey years. Significant (Kruskal-Wallis Htest: all $P<0.05)$ differences in median antibody levels were observed among parasite-specific antigens on Ambae in 2007. For P. falciparum, the anti-SE antibody level was significantly (Mann-Whitney U-test: both $P<$ 0.01 ) higher than the anti-MSP-1 $1_{19}$ and anti-AMA-1 levels. For $P$. vivax, the anti-AMA-1 antibody level was significantly (Mann-Whitney U-test: both $P<0.05$ ) higher than the anti-SE and anti-MSP1 $1_{19}$ levels.

Antibody responses were significantly correlated among all antigens, although the strength of correlation varied (Table 2). In general, the correlations were stronger between antibody responses to antigens of the same species (e.g. PfSE vs PfMSP-1 19 ) than between those of orthologous antigens (e.g. PfMSP-1 19 vs PvMSP-1 19 ). Weaker correlations were observed between antibody responses to gSG6 and the parasite antigens (Spearman's $r$ $=0.11-0.20, P<0.01$ ).

\section{Seroprevalence in the populations}

Figure 3 shows the overall seroprevalence to parasite antigens on Ambae. Among all parasite antigens, seroprevalence to crude SE was highest for both $P$. falciparum and $P$. vivax. Similar to parasite rate and other malaria indices, seroprevalence was significantly higher (Chi-square test or Fisher's exact test: all $P<$ 0.001) in 2003 than in 2007 for all parasite antigens except PvAMA-1. No significant difference was observed in seroprevalence between males and females for any of the antigens tested (Chi-square test or Fisher's exact test: all $P>0.05)$. For all parasite antigens, the proportion of seropositive individuals significantly increased with age
(Chi-square test: all $P<0.001)$. Seroprevalence to gSG6 is shown in Fig. 3b. As expected from an island without anopheline mosquitoes, all samples from Futuna were seronegative. On Ambae, a significant (Fisher's exact test: $P<0.001)$ decrease in seroprevalence to gSG6 was observed, from $39 \%$ in 2003 to $0.7 \%$ in 2007 . Nevertheless, gSG6 seroprevalence in 2003 was similar among age groups.

\section{Changes in SCR}

The relationship between seroprevalence and age on Ambae was further examined using reversible catalytic conversion models. Seroconversion curves for parasite antigens are shown in Fig. 4. As was observed for the overall seroprevalence, the SCRs for all $P$. falciparum antigens significantly decreased between 2003 and 2007 on Ambae, as evidenced by the non-overlapping confidence intervals. The resulting SCRs suggest at least a three-fold decrease in $P$. falciparum transmission on Ambae. For P. vivax, significant decreases in SCRs were observed for SE and MSP-1 19 , but not for AMA-1 between 2003 and 2007. The decreases in SCRs were smaller in magnitude for $P$. vivax antigens than their counterparts for P. falciparum.

\section{Variables associated with seropositivity}

We performed univariate and multivariate logistic regression analyses to identify factors associated with seropositivity to any $P$. falciparum and $P$. vivax specific antigens, as well as Anopheles gSG6 on Ambae Island (Table 3). In the adjusted model, the survey year 2007 was significantly associated with lower seropositivity to all antigens tested. Significant associations were found between age and seropositivity to both $P$. falciparum and $P$. vivax antigens. For $P$. falciparum antigens, the likelihood of seropositivity increased significantly with age, whereas for $P$. vivax, the increase in seropositivity was significant among adults aged $>20$ only. No association was found between age and seropositivity to the vector salivary gSG6 antigen.

Table 2 Pairwise correlation (Spearman's rank correlation coefficient) between IgG responses to parasite and mosquito antigens. All correlations were significant $(P<0.01)$

\begin{tabular}{|c|c|c|c|c|c|c|c|c|}
\hline \multirow[b]{2}{*}{ Antigen } & \multirow[b]{2}{*}{ Species } & \multicolumn{2}{|l|}{ SE } & \multicolumn{2}{|c|}{ MSP-1 $_{19}$} & \multicolumn{2}{|l|}{ AMA-1 } & \multirow{2}{*}{$\begin{array}{l}\text { gSG6 } \\
\mathrm{Ag}\end{array}$} \\
\hline & & $\overline{\mathrm{Pf}}$ & $\mathrm{PV}$ & $\mathrm{Pf}$ & $\mathrm{Pv}$ & $\overline{P f}$ & Pv & \\
\hline \multirow[t]{2}{*}{$\overline{S E}$} & Pf & & & & & & & \\
\hline & Pv & 0.5776 & & & & & & \\
\hline \multirow[t]{2}{*}{$\mathrm{MSP} 1_{19}$} & Pf & 0.7635 & 0.4741 & & & & & \\
\hline & Pv & 0.4171 & 0.6595 & 0.4325 & & & & \\
\hline \multirow[t]{2}{*}{ AMA-1 } & Pf & 0.5982 & 0.4396 & 0.6074 & 0.3595 & & & \\
\hline & Pv & 0.4367 & 0.5821 & 0.3632 & 0.465 & 0.3128 & & \\
\hline gSG6 & $\mathrm{Ag}$ & 0.1750 & 0.1934 & 0.1168 & 0.132 & 0.1937 & 0.1139 & \\
\hline
\end{tabular}



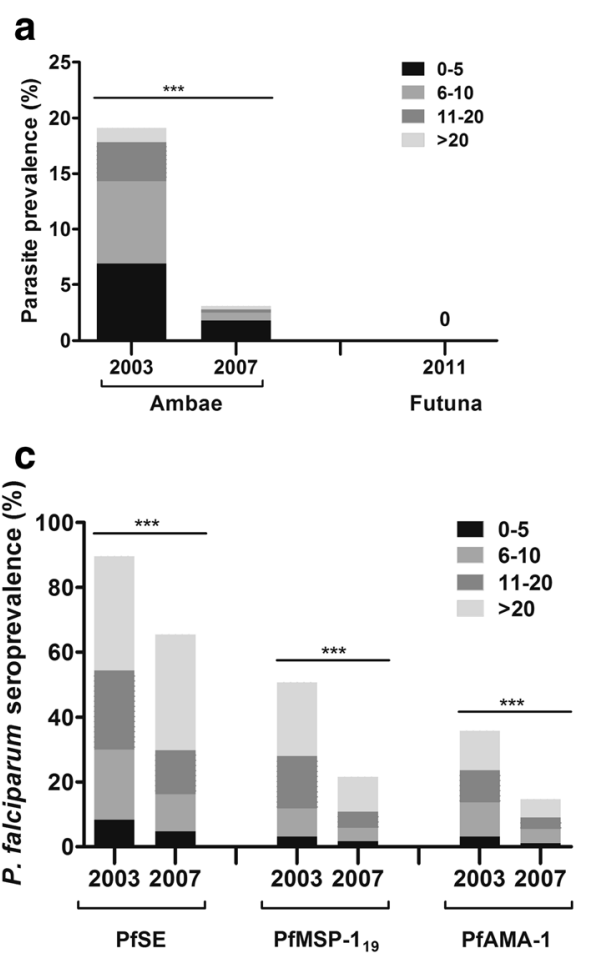

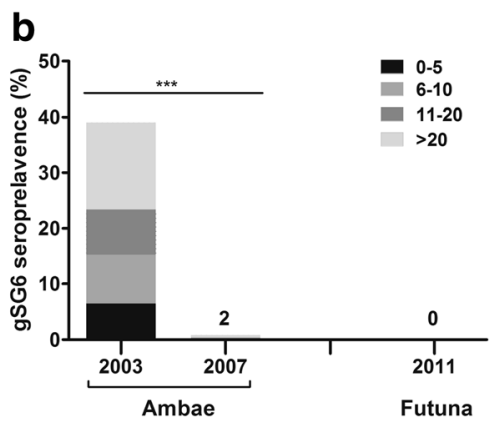

d

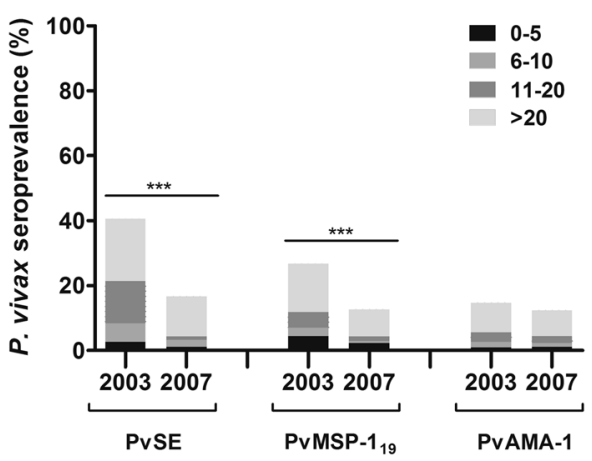

Fig. 3 Age-specific parasite prevalence by microscopy and antibody response in Ambae Island and Futuna Island. Graphs are divided by year of sampling and in four age groups for: (a) Microscopy, (b) An. gambiae salivary gland gSG6 antigen, (c) P. falciparum antigens, and (d) P. vivax antigens. Serological analyses for $P$. falciparum and $P$. vivax antigens were performed for samples from Ambae Island only. Numbers above bars show the numbers of positive individuals

\section{Discussion}

Following a gap in funding from external donors that negatively impacted ITN coverage, Vanuatu experienced a resurgence of malaria incidence in the first half of the 2000s. In 2003, Vanuatu secured financial support from the Global Fund to resume distribution of subsidised ITNs. Subsequent decreases in slide positivity rate and proportion of cases caused by $P$. falciparum at the national level were observed after 2006 (16). In this study, we examined the parasite prevalence and IgG antibody responses to antigens from the two major Plasmodium species as well as Anopheles vector on Ambae Island in 2003 and 2007, marking the peak of the resurgence and its subsidence, respectively. A significant decrease in parasite prevalence was accompanied by significant decreases in seropositivity to most parasite antigens and the vector salivary antigen, suggesting that reinforced vector control played an important role in the reduction of malaria transmission on Ambae.

The shift in species predominance on Ambae between 2003 and 2007 was consistent with the nation-wide decrease in the proportion of $P$. falciparum cases. As ITN was the main intervention tool used during the study period, the shift to $P$. falciparum- $P$. vivax co-dominance in 2007 suggests that ITNs were more protective against
P. falciparum-infected mosquitoes. Previously in Papua New Guinea, differential ITN protection was partially explained by differences in biting behaviour of An. punctulatus mosquitoes infected with different parasite species, with a higher proportion of $P$. vivax-infected mosquitoes biting humans earlier in the evening (25). Furthermore, ITNs provide no protection against $P$. vivax relapse from hypnozoites, which might explain the increase in the proportion of $P$. vivax cases in our study site as well as others, where overall malaria incidences have declined as a result of effective interventions $[6,29,30]$.

The greater decrease in $P$. falciparum prevalence between 2003 and 2007 was reflected in the more pronounced reduction in seroprevalence to $P$. falciparum antigens, especially AMA-1 (Fig. 3). The net decrease in seroprevalence to PfAMA-1 $(-0.212)$ was more than nine times higher than the net decrease in seroprevalence to PvAMA-1 (-0.023). In contrast, the net decrease in seroprevalence to crude SE was similar between $P$. falciparum (-0.244) and P. vivax (-0.239). It should be noted that crude SE contains multiple proteins, of which some are similar between parasite species [7]. Antibodies to crude SE might therefore not be species-specific, and cross-reactive antibodies might have masked the difference in exposure between $P$. falciparum and $P$. vivax. 


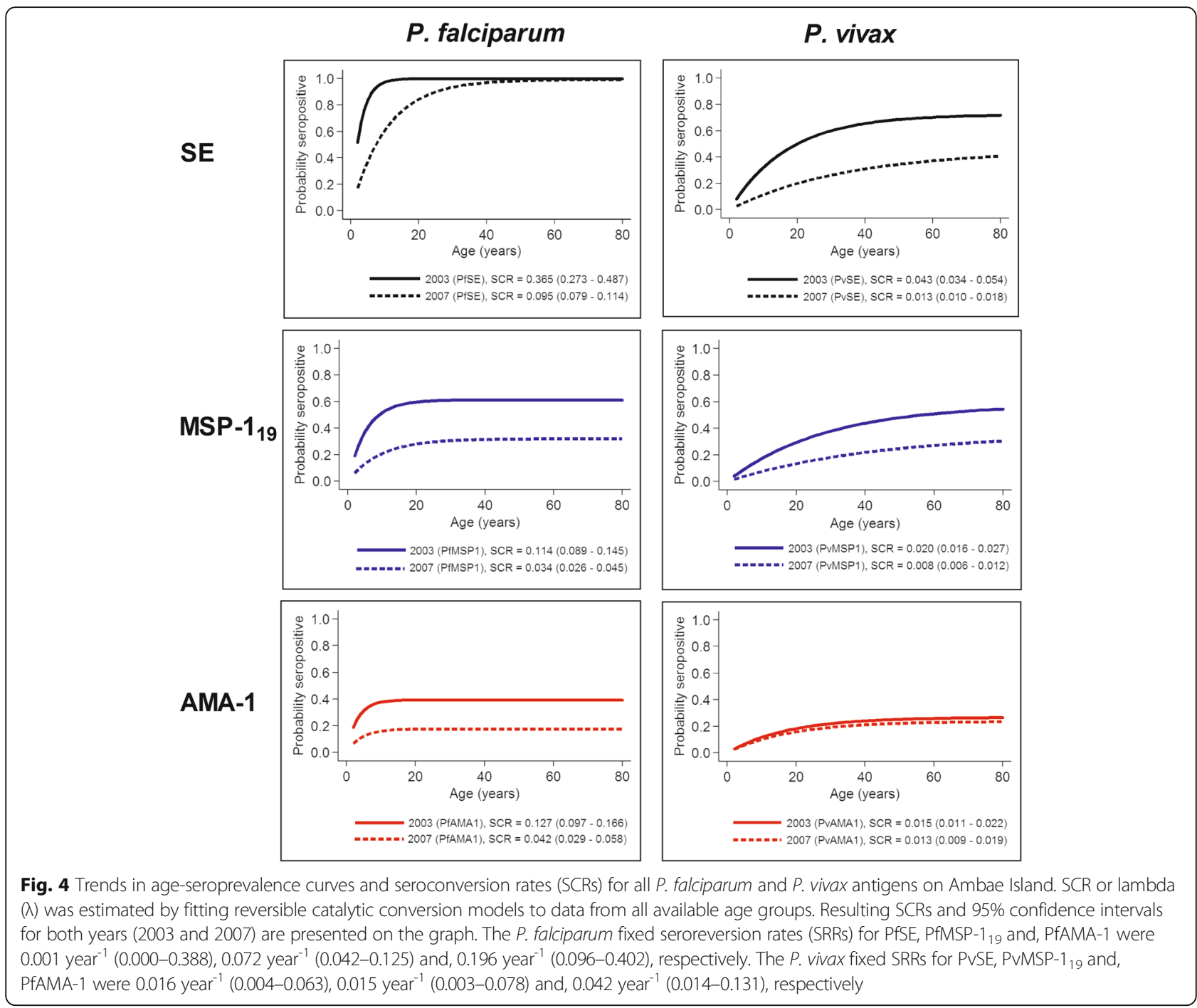

Seroprevalence reflects cumulative malaria exposure and can be used to estimate transmission intensity in a population. Modelling changes between seroprevalence and age (i.e. SCR) can help evaluate specific interventions in an area. In practice, SCR is calculated by fitting a reversible catalytic model to the age-specific malaria seroprevalence data, taking into account malaria exposure over time [28]. SCR describes the frequency per unit time (e.g. year) at which seronegative individuals becomes seropositive, and is related to the underlying force of infection [1]. Since serological data integrate exposure over time, they can reveal changes in transmission (e.g. recent outbreak) [3, 7]. In this study, statistical analyses of the seroprevalence profiles suggested that serological response generally increased with age and malaria transmission intensity decreased dramatically on Ambae between 2003 and 2007. SCRs estimated from the age-adjusted seroprevalence curves for $P$. falciparum antigens were higher than the ones for $P$. vivax antigens, reflecting the more intense transmission and the predominance of the former species on Ambae, especially in 2003 (Fig. 3). Changes in P. falciparum transmission were readily detected and the estimated decreases in transmission intensity were consistent across the three antigens used: $74.0 \%$ for PfSE (SCR from 0.365 to 0.095), $70.2 \%$ for PfMSP- $1_{19}$ (0.114 to 0.034$)$, and $66.9 \%$ for PfAMA-1 (0.127 to 0.042). For P. vivax, the decreases in transmission were more variable: $69.8 \%$ for PvSE (0.043 to $0.013), 60.0 \%$ for PvMSP- $1_{19}$ (0.020 to 0.008 ), and $13.3 \%$ for PvAMA-1 (0.015 to 0.013). It is unclear why the estimates from PvMSP- $1_{19}$ and PvAMA-1 were drastically different. The difference in SRR, subclass dependent half-life, inherent immunogenicity, and polymorphism or diversity between the two antigens may explain some of the variation in estimates [3]. Similar observations have also been reported previously [7, 31-33].

On Aneityum Island, SCRs decreased by $85.0 \%(0.04$ to 0.006) for PfSE and 93.3\% (0.03 to 0.002) for PvSE seven years after the implementation of an integrated 


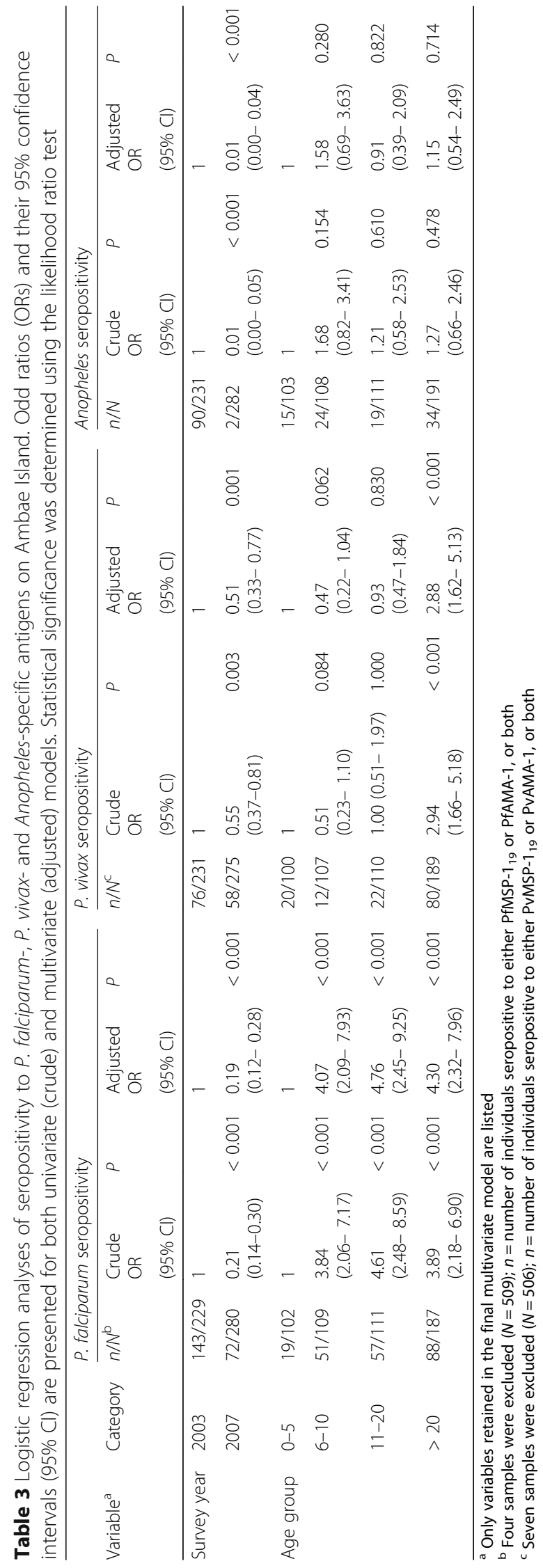


malaria elimination programme in 1991 [17]. The observed reductions on Ambae (74.0\% for PfSE and 69.8\% for PvSE) were less impressive than those on Aneityum, although the study duration on Ambae was shorter (four years). On Aneityum, seroprevalence curves revealed a distinct change in the force of infection corresponding to the abrupt cessation of transmission (19). In contrast, the decrease in transmission on Ambae appeared more gradual (Fig. 3). The absence of a step change in transmission may also be explained by the small size of our samples [34, 35]. Additional follow-up studies with samples from more recent years will be needed to confirm the longterm effect of malaria control interventions on Ambae.

Antibody response to salivary antigen gSG6 was previously shown to be a reliable indicator of human exposure to Afrotropical malaria vectors [19, 20, 36-38]. The SG6 protein first identified in An. gambiae [39], was further reported to be highly conserved among a few Anopheles species [40] and under purifying selection in an An. gambiae population from Burkina Faso [41]. Comparison of SG6 protein sequences among 16 Anopheles species [42] showed that the An. gambiae gSG6 shares high degrees of identity with orthologues from the main African (An. arabiensis, 98\%; An. funestus, 80\%), Asian (An. stephensi and An. maculatus, 79\%; An. culicifacies, 72\%; An. sinensis, 61\%; An. dirus, 54\%) and European (An. atroparvus, 66\%) malaria vectors, whereas a more limited identity (52\%) was found with An. farauti (B. Arcà et al. unpublished data). Notably, SG6 is absent in An. albimanus and An. darlingi, members of the subgenus Nyssorhynchus and important malaria vectors in Central and South America. The current study is the first to describe the use of recombinant gSG6 antigen to evaluate exposure to Anopheles bites in the Pacific, where all major malaria vectors belong to the An. punctulatus group [13]. Anopheles farauti (s.s.), the only malaria vector in Vanuatu, is absent on Futuna Island $(10,13)$. Accordingly, all participants from Futuna in this study were seronegative to gSG6 (Fig. 3b). On Ambae, exposure to Anopheles mosquito bites was greatly reduced, evidenced by the decrease in seroprevalence to gSG6 from 39\% in 2003 to $0.7 \%$ in 2007 . This reduction in vector exposure suggested the positive impact of ITN distribution after 2003 on vector population density and/or changes in vector behaviour (e.g. preference and aggressiveness towards humans). Nevertheless, a recent survey showed lower ITN use on Ambae (68\%) than on Aneityum (73\%), where elimination-specific effort has successfully halted malaria transmission [22].

Anti-gSG6 antibody response on Ambae closely followed patterns of decreases in malaria prevalence and community-level antibody responses to all parasite antigens except PvAMA-1. This finding is in agreement with previous reports of malaria incidence and Anopheles- and malaria-specific antibody responses in Africa [18, 38, 43]. Previous studies showed the utility of the gSG6 antigen in evaluating the short-term (< one year) efficacy of ITN use [44, 45]. In this study, we demonstrated that gSG6 might also be useful for long-term monitoring. As Vanuatu and neighbouring Solomon Islands embark on malaria elimination (17), serological tools to measure exposure to vectors may become more important as the standard EIR becomes more difficult to determine accurately at low transmission settings (41).

A number of caveats should be considered in this study. The most obvious one concerns the relatively small number of individuals sampled in each year. As small sample sizes might be sufficient to detect a significant reduction in SCR [35], but invariantly lead to poor estimation precision of current SCR and limit the likelihood of identifying significant changes point in malaria transmission over time for the reverse catalytic model $[34,35]$. Samples were also collected using a convenience sampling method. Although this approach is valid in obtaining an estimate of antimalarial antibody prevalence [3], it can result in an overestimation of malaria incidence in the area. While the recombinant An. gambiae gSG6 antigen could be used to measure exposure to An. farauti bites in Vanuatu, the limited conservation between the SG6 protein from these two species (52\% identity, $70 \%$ similarity) likely result in lower detection sensitivity. Serological analyses using more sensitive biomarker such as CE5 may provide a more accurate measure of exposure to vectors in a population (42). Parasite prevalence in the current study was determined solely by microscopy which likely underestimated the true prevalence. Molecular screening by PCR consistently detects at least twice as many infections as microscopy [46]. Recent studies have confirmed that in malaria-endemic areas submicroscopic (microscopy negative but PCR positive) $P$. falciparum and P. vivax infections are common $[47,48]$. The prevalence of these sub-microscopic infections and their contribution to malaria burden are age-dependent [49]. In areas of very low transmission sub-microscopic carriers are estimated to be the source of $20-50 \%$ of all human-tomosquito transmissions [46]. Considering that malaria transmission has decreased further in Vanuatu, assessing sub-microscopic parasite carriage will be critical in monitoring malaria elimination measures.

\section{Conclusions}

This study showed the decreases in malaria prevalence and antibody responses to crude SE, MSP- $1_{19}$, and AMA-1 from $P$. falciparum and $P$. vivax on Ambae Island, Vanuatu between 2003 and 2007. These decreases were matched by a reduction in seroprevalence to the Anopheles-specific salivary gSG6 antigen, suggesting that ITNs were effective in suppressing malaria transmission. This study also demonstrated the utility of recombinant 
gSG6, originally developed to measure exposure to Afrotropical vectors, in the Pacific where the An. punctulatus group is endemic. Larger follow-up surveys are required to examine more subtle changes in transmission.

\section{Abbreviations}

ACT: Artemisinin-based combination therapy; AMA-1: apical membrane antigen 1; EIR: entomological inoculation rate; ELISA: enzyme-linked immunosorbent assay; gSG6: Anopheles gambiae salivary gland antigen; ITN: insecticide-treated bed nets; LLIN: long-lasting insecticide treated nets; MDA: mass drug administration; MSP-1 19: 19 kDa merozoite surface protein1; OD: optical density; SCR: seroconversion rate; SE: crude schizont extract

\section{Acknowledgments}

We would like to extend our gratitude to the communities and community leaders for their support and participation in the surveys. We wish to sincerely thank all members in the field team.

\section{Funding}

This work (P.I.: AK) was supported by Swedish Research Council grants (5232009-3233, 348-2012-6346, and 348-2013-6311), Japan Society for Promotion of Science (JSPS) Core-to-Core Program, B. Asia-Africa Science Platforms JSPS KAKENHI grant (26257504), and the collaborative research grant of Nagasaki University Institute of Tropical Medicine. ZMI was supported by a fellowship from the Academic Training Scheme for Lecturers (SLAl) administrated by the Ministry of Higher Education, Malaysia.

\section{Availability of data and materials}

The data supporting the conclusions of this article are included within the article. The authors will make all raw data available as reasonably requested, except subjects-identifiers.

\section{Authors' contributions}

$\mathrm{ZMI}$ and $\mathrm{AK}$ designed the study. ZMI, MM, MK and KJ carried out laboratory work. ZMI performed the data analyses. ZMI, CWC, GT, BA, CD and AK interpreted the data and wrote the manuscript. All authors have read and approved the final manuscript.

\section{Competing interests}

The authors declare that they have no competing interests.

\section{Consent for publication}

Not applicable

\section{Ethics approval and consent to participate}

All subject-identifiers were removed from the data prior to extraction from database. Non-personal identifiers were used during analysis and presentation. Written informed consent was obtained from all subjects. The study was approved by the Ministry of Health in Vanuatu and by the Committee on the Ethics of Human Research of Karolinska Institutet in Sweden.

\section{Publisher's Note}

Springer Nature remains neutral with regard to jurisdictional claims in published maps and institutional affiliations.

\footnotetext{
Author details

'Island Malaria Group, Department of Microbiology, Tumor and Cell Biology, Karolinska Institutet, Stockholm, Sweden. ${ }^{2}$ Department of Parasitology and Medical Entomology, Faculty of Medicine, Universiti Kebangsaan Malaysia Medical Centre, Kuala Lumpur, Malaysia. ${ }^{3}$ Ministry of Health, Port Vila, Vanuatu. ${ }^{4}$ Department of Immunology and Infection, London School of Hygiene \& Tropical Medicine, London, UK. ${ }^{5}$ Yeast and Fermentation, Carlsberg Research Laboratory, Copenhagen, Denmark. ${ }^{6}$ Department of Public Health and Infectious Diseases, Parasitology Section, Sapienza University of Rome, Rome, Italy. ${ }^{7}$ Department of Parasitology and Research Centre for Infectious Disease Sciences, Graduate School of Medicine, Osaka City University, Osaka, Japan. ${ }^{8}$ Institute of Tropical Medicine, Nagasaki University, Nagasaki, Japan.
}

Received: 11 January 2017 Accepted: 13 April 2017

Published online: 26 April 2017

\section{References}

1. Drakeley CJ, Corran PH, Coleman PG, Tongren JE, McDonald SL, Carneiro I, et al. Estimating medium- and long-term trends in malaria transmission by using serological markers of malaria exposure. Proc Natl Acad Sci USA. 2005; 102:5108-13.

2. Bousema T, Youssef RM, Cook J, Cox J, Alegana VA, Amran J, et al. Serologic markers for detecting malaria in areas of low endemicity, Somalia, 2008. Emerg Infect Dis. 2010;16:392-9.

3. Stewart L, Gosling R, Griffin J, Gesase S, Campo J, Hashim R, et al. Rapid assessment of malaria transmission using age-specific sero-conversion rates. PLoS One. 2009:4:e6083.

4. van den Hoogen LL, Griffin JT, Cook J, Sepulveda N, Corran P, Conway DJ, et al. Serology describes a profile of declining malaria transmission in Farafenni. Gambia Malar J. 2015;14:416.

5. Bruce-Chwatt LJ, Draper CC, Avramidis D, Kazandzoglou O. Seroepidemiological surveillance of disappearing malaria in Greece. J Trop Med Hyg. 1975;78:194-200

6. Bruce-Chwatt LJ, Draper CC, Konfortion P. Seroepidemiological evidence of eradication of malaria from Mauritius. Lancet. 1973:2:547-51.

7. Cook J, Reid H, lavro J, Kuwahata M, Taleo G, Clements A, et al. Using serological measures to monitor changes in malaria transmission in Vanuatu. Malar J. 2010;9:169.

8. Zakeri S, van den Hoogen LL, Mehrizi AA, Karimi F, Raeisi A, Drakeley C. Anti-malarial seroprevalence assessment during an elimination programme in Chabahar District, south-eastern Iran. Malar J. 2016:15:382.

9. Cook J, Kleinschmidt I, Schwabe C, Nseng G, Bousema T, Corran PH, et al. Serological markers suggest heterogeneity of effectiveness of malaria control interventions on Bioko Island, equatorial Guinea. PLoS One. 2011;6: e25137.

10. Kaneko A, Taleo G, Kalkoa M, Yaviong J, Reeve PA, Ganczakowski M, et al. Malaria epidemiology, glucose 6-phosphate dehydrogenase deficiency and human settlement in the Vanuatu Archipelago. Acta Trop. 1998;70:285-302.

11. World Health Organization. World Malaria Report. Geneva: WHO; 2015.

12. Chaves LF, Kaneko A, Taleo G, Pascual M, Wilson ML. Malaria transmission pattern resilience to climatic variability is mediated by insecticide-treated nets. Malar J. 2008;7:100

13. Sinka ME, Bangs MJ, Manguin S, Chareonviriyaphap T, Patil AP, Temperley $\mathrm{WH}$, et al. The dominant Anopheles vectors of human malaria in the AsiaPacific region: occurrence data, distribution maps and bionomic precis. Parasit Vectors. 2011:4:89.

14. Kaneko A, Taleo G, Kalkoa M, Yamar S, Kobayakawa T, Bjorkman A. Malaria eradication on islands. Lancet. 2000;356:1560-4.

15. Atkinson JA, Fitzgerald L, Toaliu H, Taleo G, Tynan A, Whittaker M, et al. Community participation for malaria elimination in Tafea Province, Vanuatu: Part I. Maintaining motivation for prevention practices in the context of disappearing disease. Malar J. 2010;9:93.

16. The Pacific Malaria Initiative Survey Group (PMISG) on behalf of the Ministries of Health of Vanuatu and Solomon Islands. Malaria on isolated Melanesian islands prior to the initiation of malaria elimination activities. Malar J. 2010;9:218.

17. Kaneko A, Chaves LF, Taleo G, Kalkoa M, Isozumi R, Wickremasinghe R, et al. Characteristic age distribution of Plasmodium vivax infections after malaria elimination on Aneityum Island, Vanuatu. Infect Immun. 2014:82:243-52.

18. Poinsignon A, Cornelie S, Ba F, Boulanger D, Sow C, Rossignol M, et al. Human lgG response to a salivary peptide, gSG6-P1, as a new immunoepidemiological tool for evaluating low-level exposure to Anopheles bites. Malar J. 2009;8:198.

19. Rizzo C, Lombardo F, Ronca R, Mangano V, Sirima SB, Nebie I, et al. Differential antibody response to the Anopheles gambiae gSG6 and CE5 salivary proteins in individuals naturally exposed to bites of malaria vectors. Parasit Vectors. 2014;7:549

20. Rizzo C, Ronca R, Fiorentino G, Mangano VD, Sirima SB, Nebie I, et al. Wide cross-reactivity between Anopheles gambiae and Anopheles funestus SG6 salivary proteins supports exploitation of gSG6 as a marker of human exposure to major malaria vectors in tropical Africa. Malar J. 2011;10:206

21. Doucoure S, Drame PM. Salivary biomarkers in the control of mosquitoborne diseases. Insects. 2015;6:961-76. 
22. Watanabe N, Kaneko A, Yamar S, Leodoro H, Taleo G, Tanihata T, et al. Determinants of the use of insecticide-treated bed nets on islands of preand post-malaria elimination: an application of the health belief model in Vanuatu. Malar J. 2014;13:441.

23. Mills AR. A malaria survey of Futuna in the New Hebrides. J Trop Med Hyg 1954:57:99-107.

24. Chaves LF, Taleo G, Kalkoa M, Kaneko A. Spleen rates in children: an old and new surveillance tool for malaria elimination initiatives in island settings. Trans R Soc Trop Med Hyg. 2011;105:226-31.

25. Corran PH, Cook J, Lynch C, Leendertse H, Manjurano A, Griffin J, et al. Dried blood spots as a source of anti-malarial antibodies for epidemiological studies. Malar J. 2008;7:195.

26. Troye-Blomberg M, Perlmann $H$, Patarroyo ME, Perlmann P. Regulation of the immune response in Plasmodium falciparum malaria. II. Antigen specific proliferative responses in vitro. Clin Exp Immunol. 1983;53:345-53.

27. Ihalamulla RL, Mendis KN. Plasmodium vivax: isolation of mature asexual stages and gametocytes from infected human blood by colloidal silica (Percoll) gradient centrifugation. Trans R Soc Trop Med Hyg. 1987:81:25-8.

28. Corran P, Coleman P, Riley E, Drakeley C. Serology: a robust indicator of malaria transmission intensity? Trends Parasitol. 2007;23:575-82.

29. Mendis K, Sina BJ, Marchesini P, Carter R. The neglected burden of Plasmodium vivax malaria. Am J Trop Med Hyg. 2001;64:97-106.

30. Childs DZ, Cattadori IM, Suwonkerd W, Prajakwong S, Boots M. Spatiotemporal patterns of malaria incidence in northern Thailand. Trans $R$ Soc Trop Med Hyg. 2006;100:623-31.

31. Wong J, Hamel MJ, Drakeley CJ, Kariuki S, Shi YP, Lal AA, et al. Serological markers for monitoring historical changes in malaria transmission intensity in a highly endemic region of Western Kenya, 1994-2009. Malar J. 2014;13:451.

32. Supargiyono S, Bretscher MT, Wijayanti MA, Sutanto I, Nugraheni D, Rozqie $R$, et al. Seasonal changes in the antibody responses against Plasmodium falciparum merozoite surface antigens in areas of differing malaria endemicity in Indonesia. Malar J. 2013;12:444.

33. Cook J, Speybroeck N, Sochanta T, Somony H, Sokny M, Claes F, et al. Seroepidemiological evaluation of changes in Plasmodium falciparum and Plasmodium vivax transmission patterns over the rainy season in Cambodia. Malar J. 2012:11:86.

34. Sepulveda N, Drakeley C. Sample size determination for estimating antibody seroconversion rate under stable malaria transmission intensity. Malar J. 2015;14:141.

35. Sepulveda N, Paulino CD, Drakeley C. Sample size and power calculations for detecting changes in malaria transmission using antibody seroconversion rate. Malar J. 2015;14:529.

36. Proietti C, Verra F, Bretscher MT, Stone W, Kanoi BN, Balikagala B, et al. Influence of infection on malaria-specific antibody dynamics in a cohort exposed to intense malaria transmission in northern Uganda. Parasite Immunol. 2013;35:164-73.

37. Stone W, Bousema T, Jones S, Gesase S, Hashim R, Gosling R, et al. IgG responses to Anopheles gambiae salivary antigen gSG6 detect variation in exposure to malaria vectors and disease risk. PLoS One. 2012;7:e40170.

38. Rizzo C, Ronca R, Fiorentino G, Verra F, Mangano V, Poinsignon A, et al. Humoral response to the Anopheles gambiae salivary protein gSG6: a serological indicator of exposure to Afrotropical malaria vectors. PLoS One. 2011;6:e17980

39. Lanfrancotti A, Lombardo F, Santolamazza F, Veneri M, Castrignano T, Coluzzi M, Arca B. Novel CDNAs encoding salivary proteins from the malaria vector Anopheles gambiae. FEBS Lett. 2002;17:67-71.

40. Calvo E, Dao A, Pham VM, Ribeiro JM. An insight into the sialome of Anopheles funestus reveals an emerging pattern in anopheline salivary protein families. Insect Biochem Mol Biol. 2007;37:164-75.

41. Arca B, Struchiner CJ, Pham VM, Sferra G, Lombardo F, Pombi M, Ribeiro JM. Positive selection drives accelerated evolution of mosquito salivary genes associated with blood-feeding. Insect Mol Biol. 2014;23:122-31.

42. Neafsey DE, Waterhouse RM, Abai MR, Aganezov SS, Alekseyev MA, Allen JE, et al. Mosquito genomics. Highly evolvable malaria vectors: the genomes of 16 Anopheles mosquitoes. Science. 2015;347:1258522.

43. Remoue F, Cisse B, Ba F, Sokhna C, Herve JP, Boulanger D, Simondon F. Evaluation of the antibody response to Anopheles salivary antigens as a potential marker of risk of malaria. Trans R Soc Trop Med Hyg. 2006:100:363-70.

44. Drame PM, Poinsignon A, Besnard P, Le Mire J, Dos-Santos MA, Sow CS, et al. Human antibody response to Anopheles gambiae saliva: an immuno- epidemiological biomarker to evaluate the efficacy of insecticide-treated nets in malaria vector control. Am J Trop Med Hyg. 2010;83:115-21.

45. Drame PM, Poinsignon A, Besnard P, Cornelie S, Le Mire J, Toto JC, et al. Human antibody responses to the Anopheles salivary gSG6-P1 peptide: a novel tool for evaluating the efficacy of ITNs in malaria vector control. PLoS One. 2010;5:e15596

46. Okell LC, Bousema T, Griffin JT, Ouedraogo AL, Ghani AC, Drakeley CJ. Factors determining the occurrence of submicroscopic malaria infections and their relevance for control. Nat Commun. 2012:3:1237.

47. Steenkeste N, Rogers WO, Okell L, Jeanne I, Incardona S, Duval L, et al. Submicroscopic malaria cases and mixed malaria infection in a remote area of high malaria endemicity in Rattanakiri province, Cambodia: implication for malaria elimination. Malar J. 2010;9:108.

48. Waltmann A, Darcy AW, Harris I, Koepfli C, Lodo J, Vahi V, et al. High rates of asymptomatic, sub-microscopic Plasmodium vivax infection and disappearing Plasmodium falciparum malaria in an area of low transmission in Solomon Islands. PLoS Negl Trop Dis. 2015;9:e0003758.

49. Idris ZM, Chan CW, Kongere J, Gitaka J, Logedi J, Omar A, et al. High and heterogeneous prevalence of asymptomatic and sub-microscopic malaria infections on islands in Lake Victoria, Kenya. Sci Rep. 2016;6:36958.

\section{Submit your next manuscript to BioMed Central and we will help you at every step:}

- We accept pre-submission inquiries

- Our selector tool helps you to find the most relevant journal

- We provide round the clock customer support

- Convenient online submission

- Thorough peer review

- Inclusion in PubMed and all major indexing services

- Maximum visibility for your research

Submit your manuscript at www.biomedcentral.com/submit
) Biomed Central 